Renming Song

\title{
Sharp bounds on the density, Green function and jumping function of subordinate killed BM
}

Received: 17 February 2003 / Revised version: 23 October 2003 /

Published online: 2 January 2004 - (c) Springer-Verlag 2004

\begin{abstract}
Subordination of a killed Brownian motion in a domain $D \subset \mathbb{R}^{d}$ via an $\alpha / 2$-stable subordinator gives rise to a process $Z_{t}$ whose infinitesimal generator is $-\left(-\left.\Delta\right|_{D}\right)^{\alpha / 2}$, the fractional power of the negative Dirichlet Laplacian. In this paper we establish upper and lower estimates for the density, Green function and jumping function of $Z_{t}$ when $D$ is either a bounded $C^{1,1}$ domain or an exterior $C^{1,1}$ domain. Our estimates are sharp in the sense that the upper and lower estimates differ only by a multiplicative constant.
\end{abstract}

\section{Introduction}

Let $X_{t}$ be a $d$-dimensional Brownian motion in $\mathbb{R}^{d}$ and $T_{t}$ an $\alpha / 2$-stable subordinator starting at zero, $0<\alpha<2$. It is well known that $Y_{t}=X_{T_{t}}$ is a rotationally invariant $\alpha$-stable process whose generator is $-(-\Delta)^{\alpha / 2}$, the fractional power of the negative Laplacian. The potential theory corresponding to the process $Y$ is the Riesz potential theory of order $\alpha$.

Suppose that $D$ is a domain in $\mathbb{R}^{d}$, that is, an open connected subset of $\mathbb{R}^{d}$. We can kill the process $Y$ upon exiting $D$. The killed process $Y^{D}$ has been extensively studied in recent years and various deep properties have been obtained. For instance, when $D$ is a bounded $C^{1,1}$ domain, sharp estimates on the Green function of $Y^{D}$ were established in [4] and [14].

Let $\left.\Delta\right|_{D}$ be the Dirichlet Laplacian in $D$. The fractional power $-\left(-\left.\Delta\right|_{D}\right)^{\alpha / 2}$ of the negative Dirichlet Laplacian is a very useful object in analysis and partial differential equations, see, for instance, [19] and [16]. There is a Markov process $Z$ corresponding to $-\left(-\left.\Delta\right|_{D}\right)^{\alpha / 2}$ which can be obtained as follows: We first kill the Brownian motion $X$ at $\tau_{D}$, the first exit time of $X$ from $D$, and then we subordinate the killed Brownian motion using the $\alpha / 2$-stable subordinator $T_{t}$. Note that in comparison with $Y^{D}$ the order of killing and subordination has been reversed. For the differences between the processes $Y^{D}$ and $Z$, see [18].

Despite its importance, the process $Z$ has not been studied much. In [11], a relation between the harmonic functions of $Z$ and the classical harmonic functions in $D$

R. Song: Department of Mathematics, University of Illinois, Urbana, IL 61801, USA. e-mail: rsong@math.uiuc.edu

Mathematics Subject Classification (2000): Primary 60J45, Secondary 60J75, 31C25

Key words or phrases: Killed Brownian motions - Stable processes - Subordination - Fractional Laplacian - Transition density - Green function - Jumping function 
was established. In [13] (see also [9]) the domain of the Dirichlet form of $Z$ was identified when $D$ is a bounded smooth domain and $\alpha \neq 1$. In the recent paper [18] with Vondracek, we studied the process $Z$ in detail and established, among other things, the behaviors of the jumping function $J$ and the Green function $G_{D}$ of $Z$ when $D$ is a bounded $C^{1,1}$ domain. Recall that a bounded domain $D$ in $\mathbb{R}^{d}, d \geq 2$, is said to be a bounded $C^{1,1}$ domain if there exist positive constants $r_{0}$ and $M$ with the following property: for every $z \in \partial D$ and $r \in\left(0, r_{0}\right]$, there exist a function $\Gamma_{z}: \mathbb{R}^{d-1} \rightarrow \mathbb{R}$ satisfying the condition $\left|\nabla \Gamma_{z}(\xi)-\nabla \Gamma_{z}(\eta)\right| \leq M|\xi-\eta|$ for all $\xi, \eta \in \mathbb{R}^{d-1}$ and an orthonormal coordinate system $C S_{z}$ such that if $y=\left(y_{1}, \ldots, y_{d}\right)$ in the $C S_{z}$ coordinates, then

$$
B(z, r) \cap D=B(z, r) \cap\left\{y: y_{d}>\Gamma_{z}\left(y_{1}, \ldots, y_{d-1}\right)\right\} .
$$

A bounded domain in $\mathbb{R}^{1}$ is a finite open interval. So when speak of a bounded $C^{1,1}$ domain in $\mathbb{R}^{1}$, we mean a finite open interval. It is well known that for a bounded $C^{1,1}$ domain $D$, there exists $r_{1}>0$ depending only on $D$ such that for any $z \in \partial D$ and $r \in\left(0, r_{1}\right]$, there exist two balls $B_{1}^{z}(r)$ and $B_{2}^{z}(r)$ of radius $r$ such that $B_{1}^{z}(r) \subset D, B_{2}^{z}(r) \subset(\bar{D})^{c}$ and $\{z\}=\partial B_{1}^{z}(r) \cap \partial B_{2}^{z}(r)$. One of the main results of [18] is the following

Theorem 1.1. Suppose that $D$ is a bounded $C^{1,1}$ domain in $\mathbb{R}^{d}$ and $\alpha \in(0,2)$. Let $\rho(x)$ stand for the Euclidean distance between $x$ and the boundary $\partial D$ of $D$.

(1) There exist positive constants $C_{1}$ and $C_{2}$ such that for all $x, y \in D$,

$$
C_{1} \rho(x) \rho(y) \leq J(x, y) \leq C_{2}\left(\frac{\rho(x) \rho(y)}{|x-y|^{2}} \wedge 1\right) \frac{1}{|x-y|^{d+\alpha}}
$$

(2) If $d>\alpha$, then there exist positive constants $C_{3}$ and $C_{4}$ such that for all $x, y \in D$,

$$
C_{3} \rho(x) \rho(y) \leq G_{D}(x, y) \leq C_{4}\left(\frac{\rho(x) \rho(y)}{|x-y|^{2}} \wedge 1\right) \frac{1}{|x-y|^{d-\alpha}}
$$

The lower bounds in the theorem above are very poor when $|x-y|$ is small. One of the main purposes of this paper is to establish sharp lower bounds which differ from the upper bounds only by multiplicative constants. We are also going to establish sharp estimates on the transition density of the process $Z$.

The content of this paper is organized as follows. In Section 2 we review some preliminary results on subordinate killed Brownian motions obtained in [18]. In Section 3 we review the sharp estimates obtained in [20] and [21] of the transition density of killed Brownian in $D$ when $D$ is a bounded $C^{1,1}$ domain or an exterior $C^{1,1}$ domain in $\mathbb{R}^{d}(d \geq 3)$ and extend the sharp estimates in the bounded domain case to dimensions 1 and 2. In Section 4 we establish sharp estimates for the density, Green function and the jumping function of $Z$ when $D$ is a bounded $C^{1,1}$ domain or an exterior $C^{1,1}$ domain. 


\section{Preliminary results on subordinate killed Brownian motion}

Let $X^{1}=\left(\Omega^{1}, \mathcal{F}^{1}, \mathcal{F}_{t}^{1}, X_{t}^{1}, \theta_{t}^{1}, \mathbb{P}_{x}^{1}\right)$ be a $d$-dimensional Brownian motion in $\mathbb{R}^{d}$, and let $T^{2}=\left(\Omega^{2}, \mathcal{G}^{2}, T_{t}^{2}, \mathbb{P}^{2}\right)$ be an $\alpha / 2$-stable subordinator starting at zero, $0<\alpha<2$. We will consider both processes on the product space $\Omega=\Omega^{1} \times \Omega^{2}$. Thus we set $\mathcal{F}=\mathcal{F}^{1} \times \mathcal{G}^{2}, \mathcal{F}_{t}=\mathcal{F}_{t}^{1} \times \mathcal{G}^{2}$, and $\mathbb{P}_{x}=\mathbb{P}_{x}^{1} \times \mathbb{P}^{2}$. Moreover, we define $X_{t}(\omega)=X_{t}^{1}\left(\omega^{1}\right), T_{t}(\omega)=T_{t}^{2}\left(\omega^{2}\right)$, and $\theta_{t}(\omega)=\theta_{t}^{1}\left(\omega^{1}\right)$, where $\omega=\left(\omega^{1}, \omega^{2}\right) \in$ $\Omega$. Then $X=\left(\Omega, \mathcal{F}, \mathcal{F}_{t}, X_{t}, \theta_{t}, \mathbb{P}_{x}\right)$ is a $d$-dimensional $\mathcal{F}_{t}$-Brownian motion, and $T=\left(\Omega, \mathcal{G}, T_{t}, \mathbb{P}_{x}\right)$ is an $\alpha / 2$-stable subordinator starting at zero, independent of $X$ for every $\mathbb{P}_{x}$. From now on, all processes and random variables will be defined on $\Omega$.

Let $A_{t}=\inf \left\{s>0: T_{s} \geq t\right\}$ be the inverse of $T$. Since $\left(T_{t}\right)$ is strictly increasing, $\left(A_{t}\right)$ is continuous. Further, $A_{T_{t}}=t$ and $T_{A_{s}-} \leq s \leq T_{A_{s}}$.

We define a process $Y$ subordinate to $X$ by $Y_{t}=X_{T_{t}}$. It is well known that $Y$ is a rotationally invariant $\alpha$-stable process in $\mathbb{R}^{d}$. If $\mu_{t}$ is the distribution of $T_{t}$ (i.e., ( $\left.\mu_{t}, t \geq 0\right)$ is the one-sided $\alpha / 2$-stable convolution semigroup), and ( $P_{t}, t \geq 0$ ) the semigroup corresponding to the Brownian motion $X$, then for any nonnegative Borel function $f$ on $\mathbb{R}^{d}, \mathbb{E}_{x}\left(f\left(Y_{t}\right)\right)=\mathbb{E}_{x}\left(f\left(X_{T_{t}}\right)\right)=\mathbb{E}_{x}\left(\int_{0}^{\infty} f\left(X_{s}\right) \mu_{t}(d s)\right)=$ $\int_{0}^{\infty} P_{s} f(x) \mu_{t}(d s)$.

Let $D \subset \mathbb{R}^{d}$ be domain, and let $\tau_{D}^{Y}=\inf \left\{t>0: Y_{t} \notin D\right\}$ be the exit time of $Y$ from $D$. The process $Y$ killed upon exiting $D$ is defined by

$$
Y_{t}^{D}=\left\{\begin{array}{l}
Y_{t}, t<\tau_{D}^{Y} \\
\partial, \quad t \geq \tau_{D}^{Y}
\end{array}= \begin{cases}X_{T_{t}}, & t<\tau_{D}^{Y} \\
\partial, & t \geq \tau_{D}^{Y}\end{cases}\right.
$$

where $\partial$ is an isolated point serving as a cemetery.

Let $\tau_{D}=\inf \left\{t>0: X_{t} \notin D\right\}$ be the exit time of $X$ from $D$. The Brownian motion killed upon exiting $D$ is defined as

$$
X_{t}^{D}=\left\{\begin{array}{l}
X_{t}, t<\tau_{D} \\
\partial, \quad t \geq \tau_{D}
\end{array}\right.
$$

We define now the subordinate killed Brownian motion as the process obtained by subordinating $X^{D}$ via the $\alpha / 2$-stable subordinator $T_{t}$. More precisely, let $Z_{t}=$ $\left(X^{D}\right)_{T_{t}}, t \geq 0$. Then

$$
Z_{t}=\left\{\begin{array}{ll}
X_{T_{t}}, & T_{t}<\tau_{D} \\
\partial, & T_{t} \geq \tau_{D}
\end{array}= \begin{cases}X_{T_{t}}, & t<A_{\tau_{D}} \\
\partial, & t \geq A_{\tau_{D}}\end{cases}\right.
$$

where the last equality follows from the fact $\left\{T_{t}<\tau_{D}\right\}=\left\{t<A_{\tau_{D}}\right\}$. Note that $A_{\tau_{D}}$ is the lifetime of the process $Z$. Moreover, it holds that $A_{\tau_{D}} \leq \tau_{D}^{Y}$. Indeed, if $s<A_{\tau_{D}}$, then $T_{s}<\tau_{D}$, implying that $Y_{s}=X_{T_{s}} \in D$. Hence, $s<\tau_{D}^{Y}$. Therefore, the lifetime of $Z$ is less than or equal to the lifetime of $Y^{D}$.

For any nonnegative Borel function $f$ on $D$, let

$$
\begin{aligned}
& Q_{t} f(x)=\mathbb{E}_{x}\left[f\left(Y_{t}^{D}\right)\right]=\mathbb{E}_{x}\left[f\left(Y_{t}\right), t<\tau_{D}^{Y}\right]=\mathbb{E}_{x}\left[f\left(X_{T_{t}}\right), t<\tau_{D}^{Y}\right] \\
& R_{t} f(x)=\mathbb{E}_{x}\left[f\left(Z_{t}\right)\right]=\mathbb{E}_{x}\left[f\left(X^{D}\right)_{T_{t}}\right]=\mathbb{E}_{x}\left[f\left(X_{T_{t}}\right), t<A_{\tau_{D}}\right]
\end{aligned}
$$

Since $A_{\tau_{D}} \leq \tau_{D}^{Y}$, it follows that $R_{t} f(x) \leq Q_{t} f(x)$ for all $t \geq 0$.

The following result was established in [18]. 
Proposition 2.1. Suppose that there exists $C \in(0,1)$ such that $\mathbb{P}_{x}\left(X_{t} \in D\right) \leq C$ for every $t \in(0,1)$ and every $x \in \partial D$. Then

$$
(1-C)\left(1-R_{t} 1(x)\right) \leq 1-Q_{t} 1(x) \leq 1-R_{t} 1(x)
$$

for every $t \in(0,1)$ and every $x \in D$.

A domain $D \subset \mathbb{R}^{d}$ is said to satisfy an exterior cone condition if there exist a cone $K$ with vertex at the origin and a positive constant $r_{0}$, such that for each point $x \in \partial D$, there exist a translation and a rotation taking the cone $K$ into a cone $K_{x}$ with the vertex at $x$ such that

$$
K_{x} \cap B\left(x, r_{0}\right) \subset D^{c} \cap B\left(x, r_{0}\right) .
$$

Here $B\left(x, r_{0}\right)$ denotes the ball of radius $r_{0}$ centered at $x$. It is easy to show that the condition in Proposition 2.1 is true for a any domain $D \subset \mathbb{R}^{d}$ satisfying an exterior cone condition. It is well known that bounded $C^{1,1}$ domains and exterior $C^{1,1}$ domains satisfy the exterior cone condition.

Let $q(t, x, y)=q(t, y-x)$ be the transition density of the rotationally invariant $\alpha$-stable process $Y$. It is well known that the transition semigroup $Q_{t}$ corresponding to the killed stable process also has a density. Let $q^{D}(t, x, y)$ be this density. Let $r(t, x, y)$ be the density of $R_{t}$ and let $p^{D}(t, x, y)$ be the transition density of the killed Brownian motion $X^{D}$. The density $r(t, x, y)$ is given by the formula

$$
r(t, x, y)=\int_{0}^{\infty} p^{D}(s, x, y) \mu(t, s) d s,
$$

where $\mu(t, s)$ is the density of the one-sided $\alpha / 2$-stable convolution semigroup $\mu_{t}$. Let $G_{D}(x, y)$ and $G_{D}^{Y}(x, y)$ denote the Green functions of $Z$ and $Y^{D}$ respectively.The Green function of $Z$ is given by

$$
G_{D}(x, y)=\int_{0}^{\infty} r(t, x, y) d t=\frac{1}{\Gamma(\alpha / 2)} \int_{0}^{\infty} p^{D}(t, x, y) t^{\alpha / 2-1} d t .
$$

The following elementary result was shown in [18].

Proposition 2.2. Let $D$ be a domain in $\mathbb{R}^{d}$.

(i) The transition density $r(t, x, y)$ of $Z$ is jointly continuous in $(x, y)$ for each fixed $t$. Further, $r(t, x, y) \leq q^{D}(t, x, y)$ for all $t>0$ and all $(x, y) \in D \times D$.

(ii) When $d>\alpha$, the Green function $G_{D}(x, y)$ is finite and continuous on $D \times$ $D \backslash\{(x, x), x \in D\}$.

It is well known (see, for instance, Example 1.4.1 of [10] and (2.20) of [2]) that the Dirichlet form $\left(\mathcal{E}^{Y}, \mathcal{F}\right)$ associated with $Y$ is given by

$$
\begin{aligned}
& \mathcal{E}^{Y}(u, v)=\frac{1}{2} A(d,-\alpha) \int_{\mathbb{R}^{d}} \int_{\mathbb{R}^{d}} \frac{(u(x)-u(y))(v(x)-v(y))}{|x-y|^{d+\alpha}} d x d y \\
& \mathcal{F}=\left\{u \in L^{2}\left(\mathbb{R}^{d}\right): \int_{\mathbb{R}^{d}} \int_{\mathbb{R}^{d}} \frac{(u(x)-u(y))^{2}}{|x-y|^{d+\alpha}} d x d y<\infty\right\},
\end{aligned}
$$


where

$$
A(d,-\alpha)=\frac{\alpha \Gamma\left(\frac{d+\alpha}{2}\right)}{2^{1-\alpha} \pi^{d / 2} \Gamma\left(1-\frac{\alpha}{2}\right)} .
$$

If $D$ is a domain in $\mathbb{R}^{d}$, then the Dirichlet space on $L^{2}(D, d x)$ of the killed rotationally invariant $\alpha$-stable process $Y^{D}$ is $\left(\mathcal{E}^{Y}, \mathcal{F}^{D}\right)$ (cf. Theorem 4.4 .3 of [10]), where

$$
\mathcal{F}^{D}=\left\{f \in \mathcal{F}: f=0 \text { q.e. on } D^{c}\right\} .
$$

Here q.e. is the abbreviation for quasi-everywhere with respect to the Riesz capacity corresponding to the process $Y$. For $u, v \in \mathcal{F}^{D}, \mathcal{E}^{Y}(u, v)$ can be rewritten as

$$
\begin{aligned}
\mathcal{E}^{Y}(u, v)= & \int_{D} \int_{D}(u(x)-u(y))(v(x)-v(y)) J^{Y}(x, y) d x d y \\
& +\int_{D} u(x) v(x) \kappa^{Y}(x) d x,
\end{aligned}
$$

where

$$
\begin{aligned}
J^{Y}(x, y) & =\frac{1}{2} A(d,-\alpha)|x-y|^{-(d+\alpha)}, \\
\kappa^{Y}(x) & =A(d,-\alpha) \int_{D^{c}} \frac{1}{|x-y|^{d+\alpha}} d y .
\end{aligned}
$$

$J^{Y}$ and $\kappa^{Y}$ are called the the jumping and killing functions of $Y^{D}$ respectively.

$Z$ is a symmetric Markov process and so there is a Dirichlet form $(\mathcal{E}, D(\mathcal{E}))$ associated with $Z$. Let $P_{t}^{D}$ be the transition semigroup corresponding to the Brownian motion killed upon exiting $D$ and recall that the corresponding transition density is denoted by $p^{D}(t, x, y)$. It follows from [3] and [15] (see also [12]) that the jumping function $J(x, y)$ and the killing function $\kappa(x)$ of the process $Z$ are given by the following formulae respectively:

$$
\begin{aligned}
J(x, y) & =\int_{0}^{\infty} p^{D}(t, x, y) v(d t) \\
\kappa(x) & =\int_{0}^{\infty}\left(1-P_{t}^{D} 1(x)\right) v(d t),
\end{aligned}
$$

where

$$
v(d t)=\frac{\alpha / 2}{\Gamma(1-\alpha / 2)} t^{-\alpha / 2-1} d t
$$

is the Lévy measure of the $\alpha / 2$-stable subordinator.

It is easy to see from (2.6) that $J(x, y) \leq J^{Y}(x, y)$ for every $x, y \in D$. The following result, proven in [18], shows that the killing functions $\kappa(x)$ with $\kappa^{Y}(x)$ are comparable.

Proposition 2.3. Suppose that there exists $C \in(0,1)$ such that $\mathbb{P}_{x}\left(X_{t} \in D\right) \leq C$ for every $t \in(0,1)$ and every $x \in \partial D$. Then

$$
(1-C) \kappa(x) \leq \kappa^{Y}(x) \leq \kappa(x), \text { for every } x \in D .
$$


Remark 2.4. When $D$ is a bounded $C^{1,1}$ domain or an exterior $C^{1,1}$ domain, it follows easily from (2.5) that there exists a positive constant $C_{1}$ such that

$$
C_{1}^{-1}(\rho(x))^{-\alpha} \leq \kappa^{Y}(x) \leq C_{1}(\rho(x))^{-\alpha}, \quad x \in D .
$$

By using this and Proposition 2.3 it follows that there exists a constant $C_{2}$ such that

$$
C_{2}^{-1}(\rho(x))^{-\alpha} \leq \kappa(x) \leq C_{2}(\rho(x))^{-\alpha}, \quad x \in D .
$$

\section{Estimates on the density of killed Brownian motion}

Recall that, for any domain $D$ in $\mathbb{R}^{d}, p^{D}(t, x, y)$ stands for the transition density of killed Brownian motion in $D$. In this section we will concentrate on getting sharp estimates on $p^{D}$.

Theorem 3.1. If $D$ is a bounded $C^{1,1}$ domain in $\mathbb{R}^{d}$, then there exists a constant $C>0$ such that for any $t>0$ and any $x, y \in D$,

$$
p^{D}(t, x, y) \leq C\left(\frac{\rho(x) \rho(y)}{t} \wedge 1\right) t^{-d / 2} \exp \left(-\frac{|x-y|^{2}}{6 t}\right) .
$$

Proof. Let $\phi_{0}$ be the ground state of the Dirichlet Laplacian $-\Delta_{D}$. It is well known that when $D$ is a bounded $C^{1,1}$ domain, there exists a constant $c_{1}$ such that

$$
c_{1}^{-1} \rho(x) \leq \phi_{0}(x) \leq c_{1} \rho(x), \quad x \in D .
$$

Now we can repeat the proof of Theorem 4.6.9 of [7] to show that there exists a constant $c_{2}>0$ such that for any $t>0$ and any $x, y \in D$,

$$
p^{D}(t, x, y) \leq c_{2} t^{-(d+2) / 2} \phi_{0}(x) \phi_{0}(y) \exp \left(-\frac{|x-y|^{2}}{6 t}\right) .
$$

Combining the two displays above we get that there exists $c_{3}>0$ such that for any $t>0$ and any $x, y \in D$,

$$
p^{D}(t, x, y) \leq c_{3} t^{-(d+2) / 2} \rho(x) \rho(y) \exp \left(-\frac{|x-y|^{2}}{6 t}\right) .
$$

Now the theorem follows by combining the above with the trivial estimate

$$
p^{D}(t, x, y) \leq(2 \pi t)^{-d / 2} \exp \left(-\frac{|x-y|^{2}}{2 t}\right), \quad t>0, x, y \in D .
$$

The following result was recently established in [20].

Theorem 3.2. If $D$ is a bounded $C^{1,1}$ domain in $\mathbb{R}^{d}, d \geq 3$, then there exist positive constants $T_{0}, C_{1}$ and $C_{2}$ such that for any $t \in\left(0, T_{0}\right]$ and any $x, y \in D$,

$$
p^{D}(t, x, y) \geq C_{1}\left(\frac{\rho(x) \rho(y)}{t} \wedge 1\right) t^{-d / 2} \exp \left(-\frac{C_{2}|x-y|^{2}}{t}\right) .
$$


Remark 3.3. From Theorem 4.2.5 of [7] we know that, when $D$ is a bounded $C^{1,1}$ domain, there exists $S>0$ such that for all $t \geq S$ and $x, y \in D$,

$$
\frac{1}{2} e^{-\lambda_{0} t} \phi_{0}(x) \phi_{0}(y) \leq p^{D}(t, x, y) \leq \frac{3}{2} e^{-\lambda_{0} t} \phi_{0}(x) \phi_{0}(y),
$$

where $\lambda_{0}$ is the eigenvalue of the Dirichlet Laplacian $-\left.\Delta\right|_{D}$ corresponding to the ground state $\phi_{0}$. Therefore one can not expect the lower bound in the theorem above to be true for all $t>0$.

One of the main goals of this section is to show that the theorem above holds in dimensions 1 and 2 also. Before we do that, we establish some lemmas first.

Lemma 3.4. If $D$ is a bounded $C^{1,1}$ domain in $\mathbb{R}^{2}$, there exists $C>0$ such that for all $x, y \in D$ with $2|x-y| \geq \rho(x) \vee \rho(y)$,

$$
\frac{1}{C} \frac{\rho(x) \rho(y)}{|x-y|^{2}} \leq \tilde{G}_{D}(x, y) \leq C \frac{\rho(x) \rho(y)}{|x-y|^{2}},
$$

where $\tilde{G}_{D}$ stands for the Green function of the killed Brownian motion in D.

Proof. It follows from Theorem 6.23 of [6] that there exists $c_{1}>0$ such that for all $x, y \in D$,

$$
\frac{1}{c_{1}} \ln \left(1+\frac{\rho(x) \rho(y)}{|x-y|^{2}}\right) \leq \tilde{G}_{D}(x, y) \leq c_{1} \ln \left(1+\frac{\rho(x) \rho(y)}{|x-y|^{2}}\right) .
$$

When $x, y \in D$ satisfies $2|x-y| \geq \rho(x) \vee \rho(y)$, we have

$$
\frac{\rho(x) \rho(y)}{|x-y|^{2}} \leq 4 \text {. }
$$

Therefore in this case the inequalities in (3.1) are equivalent to

$$
\frac{1}{c_{2}} \frac{\rho(x) \rho(y)}{|x-y|^{2}} \leq \tilde{G}_{D}(x, y) \leq c_{2} \frac{\rho(x) \rho(y)}{|x-y|^{2}}
$$

for some $c_{2}>0$.

Lemma 3.5. Suppose that $D$ is a bounded $C^{1,1}$ domain in $\mathbb{R}^{d}, d=1,2$. For any $a_{1}>1$, there exist positive constants $T_{0}, C_{1}$ and $C_{2}$ depending only on $D$ and $a_{1}$ such that whenever $t \in\left(0, T_{0}\right], \rho^{2}(x) \geq a_{1}$ t and $\rho^{2}(y) \geq a_{1} t$ we have

$$
p^{D}(t, x, y) \geq \frac{C_{1}}{t^{d / 2}} \exp \left(-\frac{C_{2}|x-y|^{2}}{t}\right) .
$$

Proof. One can repeat the proof of Lemma 2.1 of [20] to get this result.

Lemma 3.6. Suppose that $D$ is a bounded $C^{1,1}$ domain in $\mathbb{R}^{d}, d=1,2$. For any $a_{1}>1$, there exist positive constants $T_{0}, C_{1}$ and $C_{2}$ depending only on $D$ and $a_{1}$ such that whenever $t \in\left(0, T_{0}\right], \rho^{2}(x) \leq a_{1} t$ and $\rho^{2}(y) \geq 16 a_{1} t$ we have

$$
p^{D}(t, x, y) \geq \frac{C_{1} \rho(x)}{t^{(d+1) / 2}} \exp \left(-\frac{C_{2}|x-y|^{2}}{t}\right) .
$$


Proof. This proof is similar to the proof of Lemma 2.2 of [20]. We first deal with the case $d=2$. Let $T_{0}=\frac{r_{1}}{16 a_{1}}$, where $r_{1}$ is the constant specified at the end of paragraph before Theorem 1.1. Given $x \in D$ such that $\rho^{2}(x) \leq a_{1} t$, let $\bar{x} \in \partial D$ be such that $|x-\bar{x}|=\rho(x)$. Let $x_{t}$ be chosen so that: $\bar{x}-x_{t}$ and $x-\bar{x}$ are co-linear, $\rho\left(x_{t}\right)=\sqrt{2 a_{1} t},\left|x-x_{t}\right| \leq \sqrt{2 a_{1} t}$. Then for any $y \in D$ satisfying $\rho^{2}(y) \geq 16 a_{1} t$,

$$
|y-x| \geq \rho(y)-\rho(x) \geq 3 \sqrt{a_{1} t} \geq \frac{3}{\sqrt{2}}\left|x-x_{t}\right| .
$$

Hence

$$
\begin{aligned}
& \left|y-x_{t}\right| \geq|y-x|-\left|x-x_{t}\right| \geq \frac{1}{2}|y-x| \\
& \left|y-x_{t}\right| \leq|x-y|+\left|x-x_{t}\right| \leq 4|x-y| .
\end{aligned}
$$

Let $y_{0} \in D$ be chosen so that: $\bar{x}-y_{0}$ and $x-\bar{x}$ are colinear, $\rho\left(y_{0}\right)=4 \sqrt{a_{1} t}$. Then $\left|x-y_{0}\right|<4 \sqrt{a_{1} t}$. Now write $u(z, s)=p^{D}(s, z, y)$ and $v(z)=\tilde{G}_{D}\left(z, y_{0}\right)$. Both $u$ and $v$ are positive solutions of the equation $\partial_{s} u=\Delta u$ in the region $\left(B\left(\bar{x}, 3.5 \sqrt{a_{1} t}\right) \cap D\right) \times(0, \infty)$ and $u(z, s)=v(z)=0$ when $z \in \partial D$. By the local comparison theorem in [8], there exists $c_{1}>0$ such that

$$
\frac{u(x, t)}{v(x)} \geq c_{1} \frac{u\left(x_{t}, t / 2\right)}{v\left(x_{t}\right)},
$$

that is,

$$
p^{D}(t, x, y) \geq c_{1} \frac{\tilde{G}_{D}\left(x, y_{0}\right)}{\tilde{G}_{D}\left(x_{t}, y_{0}\right)} p^{D}\left(\frac{t}{2}, x_{t}, y\right) .
$$

Obviously we have

$$
\begin{aligned}
& \left|x-y_{0}\right| \geq \rho\left(y_{0}\right)-\rho(x) \geq 3 \sqrt{a_{1} t}>2 \sqrt{a_{1} t}=\frac{\rho(x)}{2} \vee \frac{\rho\left(y_{0}\right)}{2} \\
& \left|x_{t}-y_{0}\right| \geq \rho\left(y_{0}\right)-\rho\left(x_{t}\right) \geq 2 \sqrt{a_{1} t}=\frac{\rho\left(x_{t}\right)}{2} \vee \frac{\rho\left(y_{0}\right)}{2} .
\end{aligned}
$$

Thus by Lemma 3.4 we have

$$
p^{D}(t, x, y) \geq c_{2} \frac{\rho(x) \rho\left(y_{0}\right)}{\left|x-y_{0}\right|^{2}} \frac{\left|x_{t}-y_{0}\right|^{2}}{\rho\left(x_{t}\right) \rho\left(y_{0}\right)} p^{D}\left(\frac{t}{2}, x_{t}, y\right) \geq c_{3} \frac{\rho(x)}{t^{1 / 2}} p^{D}\left(\frac{t}{2}, x_{t}, y\right) .
$$

Here we used the facts that $\left|x_{t}-y_{0}\right| /\left|x-y_{0}\right| \geq 1 / 2$ and $\rho\left(x_{t}\right)=\sqrt{2 a_{1} t}$. Since $\rho^{2}\left(x_{t}\right)=2 a_{1} t$ and $\rho^{2}(y) \geq 16 a_{1} t$, the previous lemma implies that

$$
p^{D}\left(\frac{t}{2}, x_{t}, y\right) \geq \frac{c_{4}}{t} \exp \left(-\frac{c_{5}\left|x_{t}-y\right|^{2}}{t}\right) \geq \frac{c_{6}}{t} \exp \left(-\frac{c_{7}|x-y|^{2}}{t}\right)
$$

where in the last inequality is due to (3.3). The proof of the lemma in the case of $d=2$ is now complete.

The proof of the case $d=1$ is similar to that of $d=2$, we only need to replace Lemma 3.4 by the exact formula for $\tilde{G}_{D}$ in this case. 
Lemma 3.7. Suppose that $D$ is a bounded $C^{1,1}$ domain in $\mathbb{R}^{d}, d=1,2$. For any $a_{2}>1$, there exist positive constants $T_{0}, C_{1}$ and $C_{2}$ depending only on $D$ and $a_{2}$ such that whenever $t \in\left(0, T_{0}\right], \rho^{2}(x) \leq a_{2} t$ and $\rho^{2}(y) \leq a_{2} t$ we have

$$
p^{D}(t, x, y) \geq \frac{C_{1} \rho(x) \rho(y)}{t^{(d+2) / 2}} \exp \left(-\frac{C_{2}|x-y|^{2}}{t}\right) .
$$

Proof. With the two lemmas above, one can now repeat the proof of Lemma 2.3 in [20] to get this result.

Now we are ready to extend Theorem 3.2 to dimensions 1 and 2 .

Theorem 3.8. If $D$ is a bounded $C^{1,1}$ domain in $\mathbb{R}^{d}, d=1,2$, then there exist positive constants $T_{0}, C_{1}$ and $C_{2}$ such that for any $t \in\left(0, T_{0}\right]$ and any $x, y \in D$,

$$
p^{D}(t, x, y) \geq C_{1}\left(\frac{\rho(x) \rho(y)}{t} \wedge 1\right) t^{-d / 2} \exp \left(-\frac{C_{2}|x-y|^{2}}{t}\right) .
$$

Proof. For any $t>0$ and $a_{1}>1$, put

$$
\begin{aligned}
& D_{1}=\left\{(x, y) \in D \times D: \rho^{2}(x) \geq a_{1} t, \rho^{2}(y) \geq a_{1} t\right\} \\
& D_{2}=\left\{(x, y) \in D \times D: \rho^{2}(x) \leq a_{1} t, \rho^{2}(y) \geq 16 a_{1} t\right\} \\
& D_{3}=\left\{(x, y) \in D \times D: \rho^{2}(x) \geq 16 a_{1} t, \rho^{2}(y) \leq a_{1} t\right\} \\
& D_{4}=\left\{(x, y) \in D \times D: \rho^{2}(x) \leq 16 a_{1} t, \rho^{2}(y) \leq 16 a_{1} t\right\},
\end{aligned}
$$

then $D \times D=D_{1} \cup D_{2} \cup D_{3} \cup D_{4}$. In $D_{1}$, we use Lemma 3.5, while in $D_{4}$ we apply Lemma 3.7. When $(x, y) \in D_{2}$ we know by Lemma 3.6 that there exist positive constants $c_{1}$ and $c_{2}$ such that

$$
p^{D}(t, x, y) \geq \frac{c_{1} \rho(x)}{t^{(d+1) / 2}} \exp \left(-\frac{c_{2}|x-y|^{2}}{t}\right) .
$$

Using the simple fact that $\rho(y) \leq \rho(x)+|x-y|$, we get that

$$
\frac{\rho(y)}{\sqrt{t}} \exp \left(-\frac{c_{2}|x-y|^{2}}{t}\right) \leq\left(\sqrt{a_{1}}+\frac{|x-y|}{\sqrt{t}}\right) \exp \left(-\frac{c_{2}|x-y|^{2}}{t}\right) \leq c_{3}
$$

for some constant $c_{3}$. Thus

$$
p^{D}(t, x, y) \geq \frac{c_{4} \rho(x) \rho(y)}{t^{(d+2) / 2}} \exp \left(-\frac{2 c_{2}|x-y|^{2}}{t}\right) .
$$

$D_{3}$ can be treated similarly.

The following result extends the above result to arbitrary finite time intervals.

Theorem 3.9. If $D$ is a bounded $C^{1,1}$ domain in $\mathbb{R}^{d}$, then for any $T>0$, there exist positive constants $C_{1}$ and $C_{2}$ such that for any $t \in(0, T]$ and any $x, y \in D$,

$$
p^{D}(t, x, y) \geq C_{1}\left(\frac{\rho(x) \rho(y)}{t} \wedge 1\right) t^{-d / 2} \exp \left(-\frac{C_{2}|x-y|^{2}}{t}\right) .
$$


Proof. We are only going to prove the result for $T=2 T_{0}$, where $T_{0}$ is the constant specified in Theorem 3.2 when $d \geq 3$ and Theorem 3.8 when $d<3$. Then we can repeat the argument to go from $2 T_{0}$ to $4 T_{0}$, and then $8 T_{0}, 16 T_{0}, \ldots$, to get the general case.

Throughout this proof, $C_{2}$ stands for the constant $C_{2}$ in Theorem 3.2 when $d \geq 3$ and for the constant $C_{2}$ in Theorem 3.8 when $d<3$. Without loss of generality we may assume that $C_{2} \geq 1 / 6$. For any $t \leq T_{0}$, by Theorems 3.2 and 3.8 we have

$$
\begin{aligned}
p^{D}(2 t, x, y)= & \int_{D} p^{D}(t, x, z) p^{D}(t, z, y) d z \\
\geq & c_{1} \int_{D}\left(\frac{\rho(x) \rho(z)}{t} \wedge 1\right) t^{-d / 2} e^{-\frac{2 C_{2}|x-z|^{2}}{t}}\left(\frac{\rho(z) \rho(y)}{t} \wedge 1\right) t^{-d / 2} \\
& e^{-\frac{2 C_{2}|z-y|^{2}}{t}} d z
\end{aligned}
$$

Putting $s=t /\left(12 C_{2}\right)$ and using the elementary fact that for any $c>1$,

$$
\frac{1}{c}\left(\frac{\rho(x) \rho(y)}{t} \wedge 1\right) \leq\left(\frac{\rho(x) \rho(y)}{c t} \wedge 1\right) \leq\left(\frac{\rho(x) \rho(y)}{t} \wedge 1\right), \quad t>0, x, y \in D,
$$

we get that

$$
\begin{aligned}
p^{D}(2 t, x, y) \geq & c_{2} \int_{D}\left(\frac{\rho(x) \rho(z)}{s} \wedge 1\right) s^{-d / 2} e^{-\frac{|x-z|^{2}}{6 s}} \\
& \times\left(\frac{\rho(z) \rho(y)}{s} \wedge 1\right) s^{-d / 2} e^{-\frac{|z-y|^{2}}{6 s}} d z \\
\geq & c_{3} \int_{D} p^{D}(s, x, z) p^{D}(s, z, y) d z=c_{3} p^{D}(2 s, x, y),
\end{aligned}
$$

where in the last inequality we used Theorem 3.1. Since $2 s=t /\left(6 C_{2}\right) \leq T_{0}$, we can apply Theorems 3.2 and 3.8 to get

$$
\begin{aligned}
p^{D}(2 t, x, y) & \geq c_{4}\left(\frac{\rho(x) \rho(y)}{2 s} \wedge 1\right)(2 s)^{-d / 2} \exp \left(-\frac{C_{2}|x-y|^{2}}{2 s}\right) \\
& \geq c_{5}\left(\frac{\rho(x) \rho(y)}{2 t} \wedge 1\right)(2 t)^{-d / 2} \exp \left(-\frac{c_{6}|x-y|^{2}}{2 t}\right),
\end{aligned}
$$

where in the last inequality we used (3.4).

A domain $D$ in $\mathbb{R}^{d}(d \geq 2)$ is said to be an exterior domain if its complement is a compact set. An exterior domain is said to be an exterior $C^{1,1}$ domain if there exist positive constants $r_{0}$ and $M$ with the following property: for every $z \in \partial D$ and $r \in\left(0, r_{0}\right]$, there exist a function $\Gamma_{z}: \mathbb{R}^{d-1} \rightarrow \mathbb{R}$ satisfying the condition $\left|\nabla \Gamma_{z}(\xi)-\nabla \Gamma_{z}(\eta)\right| \leq M|\xi-\eta|$ for all $\xi, \eta \in \mathbb{R}^{d-1}$ and an orthonormal coordinate system $C S_{z}$ such that if $y=\left(y_{1}, \ldots, y_{d}\right)$ in the $C S_{z}$ coordinates, then

$$
B(z, r) \cap D=B(z, r) \cap\left\{y: y_{d}>\Gamma_{z}\left(y_{1}, \ldots, y_{d-1}\right)\right\} .
$$

For exterior $C^{1,1}$ domains we have the following result. 
Theorem 3.10. If $D$ is an exterior $C^{1,1}$ domain in $\mathbb{R}^{d}, d \geq 3$, then there exist positive constants $C_{1}, C_{2}, C_{3}$ and $C_{4}$ such that for any $t>0$ and any $x, y \in D$,

$$
\begin{aligned}
C_{1}\left(\frac{\rho(x) \rho(y)}{t \wedge 1} \wedge 1\right) t^{-d / 2} e^{-\frac{C_{2}|x-y|^{2}}{t}} & \leq p^{D}(t, x, y) \\
& \leq C_{3}\left(\frac{\rho(x) \rho(y)}{t \wedge 1} \wedge 1\right) t^{-d / 2} e^{-\frac{C_{4}|x-y|^{2}}{t}} .
\end{aligned}
$$

Proof. It follows from Theorem 1.1 of [21] that there exist positive constants $c_{1}$, $c_{2}, c_{3}$ and $c_{4}$ such that for any $t>0$ and any $x, y \in D$,

$$
\begin{aligned}
& c_{1}\left(\frac{\rho(x)}{\sqrt{t} \wedge 1} \wedge 1\right)\left(\frac{\rho(y)}{\sqrt{t} \wedge 1} \wedge 1\right) t^{-d / 2} e^{-\frac{c_{2}|x-y|^{2}}{t}} \\
& \quad \leq p^{D}(t, x, y) \leq c_{3}\left(\frac{\rho(x)}{\sqrt{t} \wedge 1} \wedge 1\right)\left(\frac{\rho(y)}{\sqrt{t} \wedge 1} \wedge 1\right) t^{-d / 2} e^{-\frac{c_{4}|x-y|^{2}}{t}} .
\end{aligned}
$$

So the upper bound in the theorem follows immediately.

When $\rho(x) \vee \rho(y) \leq \sqrt{t} \wedge 1$, or when $\rho(x) \wedge \rho(y) \geq \sqrt{t} \wedge 1$, we have

$$
\left(\frac{\rho(x)}{\sqrt{t} \wedge 1} \wedge 1\right)\left(\frac{\rho(y)}{\sqrt{t} \wedge 1} \wedge 1\right)=\left(\frac{\rho(x) \rho(y)}{t \wedge 1} \wedge 1\right) .
$$

If $\rho(x) \leq \sqrt{t} \wedge 1$ and $\rho(y) \geq \sqrt{t} \wedge 1$, then using the inequality $\rho(y) \leq \rho(x)+|x-y|$ and the fact that for any $c>0$ the function $s e^{-c s^{2}}$ is bounded on $(0, \infty)$ we see that

$$
\begin{aligned}
& c_{1}\left(\frac{\rho(x)}{\sqrt{t} \wedge 1} \wedge 1\right)\left(\frac{\rho(y)}{\sqrt{t} \wedge 1} \wedge 1\right) t^{-d / 2} e^{-\frac{c_{2}|x-y|^{2}}{t}} \\
& \quad=c_{1} \frac{\rho(x)}{\sqrt{t} \wedge 1} t^{-d / 2} e^{-\frac{c_{2}|x-y|^{2}}{t}} \\
& \quad \geq c_{5} \frac{\rho(x)}{\sqrt{t} \wedge 1} \frac{\rho(y)}{\sqrt{t} \wedge 1} e^{-\frac{c_{2}|x-y|^{2}}{t}} t^{-d / 2} e^{-\frac{c_{2}|x-y|^{2}}{t}} \\
& \quad=c_{5} \frac{\rho(x) \rho(y)}{t \wedge 1} t^{-d / 2} e^{-\frac{2 c_{2}|x-y|^{2}}{t}} \\
& \geq c_{5}\left(\frac{\rho(x) \rho(y)}{t \wedge 1} \wedge 1\right) t^{-d / 2} e^{-\frac{2 c_{2}|x-y|^{2}}{t}} .
\end{aligned}
$$

The proof is now complete.

\section{Sharp bounds on the density, Green function and the jumping kernel}

We start this section with sharp estimates on the Green function and jumping function in the case of when $D$ is a bounded $C^{1,1}$ domain.

Theorem 4.1. Suppose that $D$ is a bounded $C^{1,1}$ domain in $\mathbb{R}^{d}$ and $\alpha \in(0,2)$. Let $J$ and $G_{D}$ stand for the jumping function and Green function of $Z$ respectively. 
(1) There exist positive constants $C_{1}$ and $C_{2}$ such that for all $x, y \in D$,

$$
\begin{aligned}
C_{1}\left(\frac{\rho(x) \rho(y)}{|x-y|^{2}} \wedge 1\right) \frac{1}{|x-y|^{d+\alpha}} & \leq J(x, y) \\
& \leq C_{2}\left(\frac{\rho(x) \rho(y)}{|x-y|^{2}} \wedge 1\right) \frac{1}{|x-y|^{d+\alpha}}
\end{aligned}
$$

(2) If $d>\alpha$, then there exist positive constants $C_{3}$ and $C_{4}$ such that for all $x, y \in D$,

$$
\begin{aligned}
C_{3}\left(\frac{\rho(x) \rho(y)}{|x-y|^{2}} \wedge 1\right) \frac{1}{|x-y|^{d-\alpha}} & \leq G_{D}(x, y) \\
& \leq C_{4}\left(\frac{\rho(x) \rho(y)}{|x-y|^{2}} \wedge 1\right) \frac{1}{|x-y|^{d-\alpha}}
\end{aligned}
$$

Proof. The upper bounds were established in [18], so we only need to prove the lower bounds. The proofs of the lower bounds for $J$ and $G_{D}$ are similar, we are only going to write down the details for $G_{D}$. It follows from (2.3) that

$$
G_{D}(x, y)=\frac{1}{\Gamma(\alpha / 2)} \int_{0}^{\infty} p^{D}(t, x, y) t^{\alpha / 2-1} d t
$$

Thus it follows from Theorem 3.9 that for any $T>0$ there exist positive constant $c_{1}$ and $c_{2}$ such that

$$
G_{D}(x, y) \geq \frac{c_{1}}{\Gamma(\alpha / 2)} \int_{0}^{T}\left(\frac{\rho(x) \rho(y)}{t} \wedge 1\right) t^{-\frac{d}{2}-1+\frac{\alpha}{2}} e^{-\frac{c_{2}|x-y|^{2}}{t}} d t .
$$

We may and do assume that $x \neq y$. Let $R$ be the diameter of the domain $D$. Without loss of generality we may assume that $R^{2}=T$. Then for any $x, y \in D$, we have $\rho(x) \rho(y)<R^{2}=T$. We now prove the lower bound by dealing with 2 separate cases.

(i) $\frac{|x-y|^{2}}{\rho(x) \rho(y)}<2$. In this case we have

$$
\begin{aligned}
G_{D}(x, y) & \geq \frac{c_{1}}{\Gamma(\alpha / 2)} \int_{0}^{\rho(x) \rho(y)} t^{-\frac{d}{2}-1+\frac{\alpha}{2}} e^{-\frac{c_{2}|x-y|^{2}}{t}} d t \\
& =\frac{c_{1} c_{2}^{-(d-\alpha) / 2}}{\Gamma(\alpha / 2)} \frac{1}{|x-y|^{d-\alpha}} \int_{\frac{c_{2}|x-y|^{2}}{\rho(x) \rho(y)}}^{\infty} s^{(d-\alpha) / 2-1} e^{-s} d s \\
& \geq \frac{c_{1} c_{2}^{-(d-\alpha) / 2}}{\Gamma(\alpha / 2)} \frac{1}{|x-y|^{d-\alpha}} \int_{2 c_{2}}^{\infty} s^{(d-\alpha) / 2-1} e^{-s} d s \\
& =\frac{c_{1} c_{4} c_{2}^{-(d-\alpha) / 2}}{\Gamma(\alpha / 2)} \frac{1}{|x-y|^{d-\alpha}} .
\end{aligned}
$$

(ii) $\frac{|x-y|^{2}}{\rho(x) \rho(y)} \geq 2$. In this case we have 


$$
\begin{aligned}
G_{D}(x, y) & \geq \frac{c_{1} \rho(x) \rho(y)}{\Gamma(\alpha / 2)} \int_{\rho(x) \rho(y)}^{T} t^{-\frac{d}{2}-2+\frac{\alpha}{2}} e^{-\frac{c_{2}|x-y|^{2}}{t}} d t \\
& =\frac{c_{1} c_{2}^{-(d-\alpha) / 2-1}}{\Gamma(\alpha / 2)} \frac{\rho(x) \rho(y)}{|x-y|^{d+2-\alpha}} \int_{\frac{c_{2}|x-y|^{2}}{T}}^{\frac{c_{2}|x-y|^{2}}{\rho(x) \rho(y)}} s^{(d-\alpha) / 2} e^{-s} d s \\
& \geq \frac{c_{1} c_{2}^{-(d-\alpha) / 2-1}}{\Gamma(\alpha / 2)} \frac{\rho(x) \rho(y)}{|x-y|^{d+2-\alpha}} \int_{c_{2}}^{2 c_{2}} s^{(d-\alpha) / 2} e^{-s} d s \\
& =\frac{c_{1} c_{5} c_{2}^{-(d-\alpha) / 2-1}}{\Gamma(\alpha / 2)} \frac{\rho(x) \rho(y)}{|x-y|^{d+2-\alpha}} .
\end{aligned}
$$

The proof is now complete.

As a consequence of this result, we immediately get the following very useful $3 \mathrm{G}$ inequality. For an example of an application of this inequality, see [5].

Corollary 4.2. Suppose that $D$ is a bounded $C^{1,1}$ domain in $\mathbb{R}^{d}$ and $\alpha \in(0,2)$. If $d>\alpha$, then there exists a constant $C>0$ such that

$$
\frac{G_{D}(x, y) G_{D}(y, z)}{G_{D}(x, z)} \leq C\left(\frac{\rho(y)}{\rho(x)} G_{D}(x, y)+\frac{\rho(y)}{\rho(z)} G_{D}(y, z)\right), \quad x, y, z \in D .
$$

Proof. See the proof of Proposition 4.2 of [5].

Theorem 4.3. If $D$ is an exterior $C^{1,1}$ domain in $\mathbb{R}^{d}, d \geq 3$, then there exist positive constants $C_{1}, C_{2}, C_{3}$ and $C_{4}$ such that for all $x, y \in D$,

$$
\begin{aligned}
C_{1}\left(\frac{\rho(x) \rho(y)}{|x-y|^{2} \wedge 1} \wedge 1\right) \frac{1}{|x-y|^{d+\alpha}} & \leq J(x, y) \\
& \leq C_{2}\left(\frac{\rho(x) \rho(y)}{|x-y|^{2} \wedge 1} \wedge 1\right) \frac{1}{|x-y|^{d+\alpha}} \\
C_{3}\left(\frac{\rho(x) \rho(y)}{|x-y|^{2} \wedge 1} \wedge 1\right) \frac{1}{|x-y|^{d-\alpha}} & \leq G_{D}(x, y) \\
& \leq C_{4}\left(\frac{\rho(x) \rho(y)}{|x-y|^{2} \wedge 1} \wedge 1\right) \frac{1}{|x-y|^{d-\alpha}}
\end{aligned}
$$

Proof. The proofs for $J$ and $G_{D}$ are similar, and so we only spell out the details for $G_{D}$. It follows from (2.3) that

$$
G_{D}(x, y)=\frac{1}{\Gamma(\alpha / 2)} \int_{0}^{\infty} p^{D}(t, x, y) t^{\alpha / 2-1} d t .
$$

From this and the trivial bound $p^{D}(t, x, y) \leq(4 \pi t)^{-d / 2} e^{-|x-y|^{2} /(4 t)}$ we can immediately get that there is a $c_{1}>0$ such that

$$
G_{D}(x, y) \leq \frac{c_{1}}{|x-y|^{d-\alpha}}, \quad x, y \in D
$$


From the upper bound in Theorem 3.10 we get that

$$
G_{D}(x, y) \leq \frac{C_{3}}{\Gamma(\alpha / 2)} \int_{0}^{\infty}\left(\frac{\rho(x) \rho(y)}{t \wedge 1} \wedge 1\right) t^{-\frac{d}{2}-1+\frac{\alpha}{2}} e^{-\frac{C_{4}|x-y|^{2}}{t}} d t
$$

where $C_{3}$ and $C_{4}$ are the constants in Theorem 3.10. Therefore we have for all $x, y \in D, G_{D}(x, y)$ is bounded from above by

$$
\begin{aligned}
& \frac{C_{3}}{\Gamma(\alpha / 2)} \int_{0}^{1} \rho(x) \rho(y) t^{-\frac{d}{2}-2+\frac{\alpha}{2}} e^{-\frac{C_{4}|x-y|^{2}}{t}} d t \\
& \quad+\frac{C_{3}}{\Gamma(\alpha / 2)} \int_{1}^{\infty} \rho(x) \rho(y) t^{-\frac{d}{2}-1+\frac{\alpha}{2}} e^{-\frac{C_{4}|x-y|^{2}}{t}} d t \\
& \leq \frac{C_{3}}{\Gamma(\alpha / 2)} \rho(x) \rho(y) \int_{0}^{\infty} t^{-\frac{d}{2}-2+\frac{\alpha}{2}} e^{-\frac{C_{4}|x-y|^{2}}{t}} d t \\
& \quad+\frac{C_{3}}{\Gamma(\alpha / 2)} \rho(x) \rho(y) \int_{0}^{\infty} t^{-\frac{d}{2}-1+\frac{\alpha}{2}} e^{-\frac{C_{4}|x-y|^{2}}{t}} d t \\
& \leq \frac{c_{2} \rho(x) \rho(y)}{|x-y|^{d+2-\alpha}}+\frac{c_{3} \rho(x) \rho(y)}{|x-y|^{d-\alpha}} \leq c_{4} \frac{\rho(x) \rho(y)}{|x-y|^{2} \wedge 1} \frac{1}{|x-y|^{d-\alpha}} .
\end{aligned}
$$

The proof of the upper bound is now complete.

Now we prove the lower bound. It follows from the lower bound in Theorem 3.10 that

$$
G_{D}(x, y) \geq \frac{C_{1}}{\Gamma(\alpha / 2)} \int_{0}^{\infty}\left(\frac{\rho(x) \rho(y)}{t \wedge 1} \wedge 1\right) t^{-\frac{d}{2}-1+\frac{\alpha}{2}} e^{-\frac{C_{2}|x-y|^{2}}{t}} d t
$$

where $C_{1}$ and $C_{2}$ are the constants in Theorem 3.10. We consider the following 4 separate cases.

(i) $\rho(x) \rho(y) \geq 1$. In this case we have

$$
G_{D}(x, y) \geq \frac{C_{1}}{\Gamma(\alpha / 2)} \int_{0}^{\infty} t^{-\frac{d}{2}-1+\frac{\alpha}{2}} e^{-\frac{C_{2}|x-y|^{2}}{t}} d t=\frac{c_{5}}{|x-y|^{d-\alpha}} .
$$

(ii) $\rho(x) \rho(y)<1$ and $|x-y|^{2} \leq 2 \rho(x) \rho(y)$. In this case we have

$$
\begin{aligned}
G_{D}(x, y) & \geq \frac{C_{1}}{\Gamma(\alpha / 2)} \int_{0}^{\rho(x) \rho(y)} t^{-\frac{d}{2}-1+\frac{\alpha}{2}} e^{-\frac{C_{2}|x-y|^{2}}{t}} d t \\
& =\frac{c_{6}}{|x-y|^{d-\alpha}} \int_{\frac{C_{2}|x-y|^{2}}{\rho(x) \rho(y)}}^{\infty} s^{\frac{d-\alpha}{2}-1} e^{-s} d s \\
& \geq \frac{c_{6}}{|x-y|^{d-\alpha}} \int_{2 C_{2}}^{\infty} s^{\frac{d-\alpha}{2}-1} e^{-s} d s \geq \frac{c_{7}}{|x-y|^{d-\alpha}} .
\end{aligned}
$$


(iii) $\rho(x) \rho(y)<1,|x-y|^{2} \geq 2 \rho(x) \rho(y)$ and $|x-y|^{2} \leq 1$. In this case we have

$$
\begin{aligned}
G_{D}(x, y) & \geq \frac{C_{1}}{\Gamma(\alpha / 2)} \int_{\rho(x) \rho(y)}^{1} \frac{\rho(x) \rho(y)}{t} t^{-\frac{d}{2}-1+\frac{\alpha}{2}} e^{-\frac{C_{2}|x-y|^{2}}{t}} d t \\
& =\frac{c_{8} \rho(x) \rho(y)}{|x-y|^{d-\alpha}} \int_{C_{2}|x-y|^{2}}^{\frac{C_{2}|x-y|^{2}}{\rho(x) \rho(y)}} s^{\frac{d-\alpha}{2}} e^{-s} d s \\
& \geq \frac{c_{8} \rho(x) \rho(y)}{|x-y|^{d+2-\alpha}} \int_{C_{2}}^{2 C_{2}} s^{\frac{d-\alpha}{2}} e^{-s} d s \\
& \geq \frac{c_{9} \rho(x) \rho(y)}{|x-y|^{d+2-\alpha}}=c_{9} \frac{\rho(x) \rho(y)}{|x-y|^{2} \wedge 1} \frac{1}{|x-y|^{d-\alpha}} .
\end{aligned}
$$

(iv) $\rho(x) \rho(y)<1,|x-y|^{2} \geq 2 \rho(x) \rho(y)$ and $|x-y|^{2} \geq 1$. In this case we have

$$
\begin{aligned}
G_{D}(x, y) & \geq \frac{C_{1}}{\Gamma(\alpha / 2)} \int_{1}^{\infty} \rho(x) \rho(y) t^{-\frac{d}{2}-1+\frac{\alpha}{2}} e^{-\frac{C_{2}|x-y|^{2}}{t}} d t \\
& =\frac{c_{10} \rho(x) \rho(y)}{|x-y|^{d-\alpha}} \int_{0}^{C_{2}|x-y|^{2}} s^{\frac{d-\alpha}{2}-1} e^{-s} d s \\
& \geq \frac{c_{10} \rho(x) \rho(y)}{|x-y|^{d-\alpha}} \int_{0}^{C_{2}} s^{\frac{d-\alpha}{2}-1} e^{-s} d s \\
& \geq \frac{c_{11} \rho(x) \rho(y)}{|x-y|^{d-\alpha}}=c_{11} \frac{\rho(x) \rho(y)}{|x-y|^{2} \wedge 1} \frac{1}{|x-y|^{d-\alpha}}
\end{aligned}
$$

The proof is now complete.

Similar to Corollary 4.2, we have the following

Corollary 4.4. If $D$ is an exterior $C^{1,1}$ domain in $\mathbb{R}^{d}, d \geq 3$, then there exists a constant $C>0$ such that

$$
\frac{G_{D}(x, y) G_{D}(y, z)}{G_{D}(x, z)} \leq C\left(\frac{\rho(y)}{\rho(x)} G_{D}(x, y)+\frac{\rho(y)}{\rho(z)} G_{D}(y, z)\right), \quad x, y, z \in D .
$$

By using the same argument as in the proof of Theorem 4.3, we get the following sharp estimates for the Green function $\tilde{G}_{D}$ of killed Brownian motions in exterior $C^{1,1}$ domains. As far as we know, these estimates are new.

Theorem 4.5. If $D$ is an exterior $C^{1,1}$ domain in $\mathbb{R}^{d}, d \geq 3$, then there exist positive constants $C_{1}$ and $C_{2}$ such that for all $x, y \in D$,

$$
\begin{aligned}
C_{1}\left(\frac{\rho(x) \rho(y)}{|x-y|^{2} \wedge 1} \wedge 1\right) \frac{1}{|x-y|^{d-2}} & \leq \tilde{G}_{D}(x, y) \\
& \leq C_{2}\left(\frac{\rho(x) \rho(y)}{|x-y|^{2} \wedge 1} \wedge 1\right) \frac{1}{|x-y|^{d-2}} .
\end{aligned}
$$

Proof. The proof is similar to that of Theorem 4.3. We omit the details. 
Now we deal with estimates on the density of $r(t, x, y)$ of $Z$. We start with the case of exterior $C^{1,1}$ domains.

Theorem 4.6. If $D$ is an exterior $C^{1,1}$ domain in $\mathbb{R}^{d}, d \geq 3$, then there exist positive constants $C_{1}$ and $C_{2}$ such that

$$
\begin{aligned}
& C_{1}\left(\frac{\rho(x) \rho(y)}{\left(t^{2 / \alpha}+|x-y|^{2}\right) \wedge 1} \wedge 1\right) t^{-\frac{d}{\alpha}}\left(1+\frac{|x-y|^{2}}{t^{2 / \alpha}}\right)^{-\frac{d+\alpha}{2}} \leq r(t, x, y) \\
& \leq C_{2}\left(\frac{\rho(x) \rho(y)}{\left(t^{2 / \alpha}+|x-y|^{2}\right) \wedge 1} \wedge 1\right) t^{-\frac{d}{\alpha}}\left(1+\frac{|x-y|^{2}}{t^{2 / \alpha}}\right)^{-\frac{d+\alpha}{2}} .
\end{aligned}
$$

Proof. It is easy to see that the function on $(0, \infty) \times \mathbb{R}^{d}$ defined by

$$
(t, \xi) \mapsto \int_{0}^{\infty} s^{-d / 2} e^{-\frac{|\xi|^{2}}{s}} \mu(t, s) d s
$$

is comparable to the transition density $q(t, \xi)$ of the rotationally invariant $\alpha$-stable process $Y$ on $\mathbb{R}^{d}$. Thus it follows from scaling and Theorem 2.1 of [1] that there exist positive constants $c_{1}$ and $c_{2}$ such that for all $\xi \in \mathbb{R}^{d}$,

$c_{1} t^{-\frac{d}{\alpha}}\left(1+\frac{|\xi|^{2}}{t^{2 / \alpha}}\right)^{-\frac{d+\alpha}{2}} \leq \int_{0}^{\infty} s^{-d / 2} e^{-\frac{|\xi|^{2}}{s}} \mu(t, s) d s \leq c_{2} t^{-\frac{d}{\alpha}}\left(1+\frac{|\xi|^{2}}{t^{2 / \alpha}}\right)^{-\frac{d+\alpha}{2}}$

From this and the trivial bound $p^{D}(s, x, y) \leq(4 \pi s)^{-d / 2} e^{-|x-y|^{2} /(4 s)}$ we immediately get that

$r(t, x, y)=\int_{0}^{\infty} p^{D}(s, x, y) \mu(t, s) d s \leq c_{3} t^{-\frac{d}{\alpha}}\left(1+\frac{|x-y|^{2}}{t^{2 / \alpha}}\right)^{-\frac{d+\alpha}{2}}, x, y \in D$,

for some $c_{3}>0$. Using the upper bound in Theorem 3.10 we get that for all $x, y \in D$,

$$
r(t, x, y) \leq C_{3} \int_{0}^{\infty}\left(\frac{\rho(x) \rho(y)}{s \wedge 1} \wedge 1\right) s^{-d / 2} e^{-\frac{C_{4}|x-y|^{2}}{s}} \mu(t, s) d s,
$$

where $C_{3}$ and $C_{4}$ are the constants in Theorem 3.10. Thus we have

$$
\begin{aligned}
r(t, x, y) \leq & C_{3} \int_{0}^{1} \frac{\rho(x) \rho(y)}{s} s^{-d / 2} e^{-\frac{C_{4}|x-y|^{2}}{s}} \mu(t, s) d s \\
& +C_{3} \int_{1}^{\infty} \rho(x) \rho(y) s^{-d / 2} e^{-\frac{C_{4}|x-y|^{2}}{s}} \mu(t, s) d s \\
\leq & C_{3} \rho(x) \rho(y) \int_{0}^{\infty} s^{-(d+2) / 2} e^{-\frac{C_{4}|x-y|^{2}}{s}} \mu(t, s) d s
\end{aligned}
$$




$$
\begin{aligned}
& +C_{3} \rho(x) \rho(y) \int_{0}^{\infty} s^{-d / 2} e^{-\frac{C_{4}|x-y|^{2}}{s}} \mu(t, s) d s \\
\leq & c_{4} \rho(x) \rho(y) t^{-\frac{d+2}{\alpha}}\left(1+\frac{|x-y|^{2}}{t^{2 / \alpha}}\right)^{-\frac{d+2+\alpha}{2}} \\
& +c_{5} \rho(x) \rho(y) t^{-\frac{d}{\alpha}}\left(1+\frac{|x-y|^{2}}{t^{2 / \alpha}}\right)^{-\frac{d+\alpha}{2}} \\
\leq & c_{6} \frac{\rho(x) \rho(y)}{\left(t^{2 / \alpha}+|x-y|^{2}\right) \wedge 1} t^{-\frac{d}{\alpha}}\left(1+\frac{|x-y|^{2}}{t^{2 / \alpha}}\right)^{-\frac{d+\alpha}{2}} .
\end{aligned}
$$

The proof of the upper bound is now finished.

Now we deal with the lower bound. It follows from Section 2.4 of [22] that there exists $M>1$ such that

$$
c_{7} u^{-\frac{\alpha}{2}-1} \leq \mu(1, u) \leq c_{8} u^{-\frac{\alpha}{2}-1}, \quad u \geq M
$$

for some positive constants $c_{7}$ and $c_{8}$. In the remainder of this proof, $C_{1}$ and $C_{2}$ stands for the constant $C_{1}$ and $C_{2}$ in Theorem 3.10 respectively. We are going to prove the lower bound in the following separate cases.

(i) $\rho(x) \rho(y) \geq 1$. In this case we have

$$
\begin{aligned}
r(t, x, y) & \geq C_{1} \int_{0}^{\infty} s^{-d / 2} e^{-\frac{C_{2}|x-y|^{2}}{s}} \mu(t, s) d s \\
& \geq c_{9} t^{-\frac{d}{\alpha}}\left(1+\frac{|x-y|^{2}}{t^{2 / \alpha}}\right)^{-\frac{d+\alpha}{2}}
\end{aligned}
$$

for some $c_{9}>0$.

(ii) $\rho(x) \rho(y)<1,|x-y|^{2} \leq \rho(x) \rho(y)$ and $\frac{|x-y|^{2}}{t^{2 / \alpha}} \geq 2 M$. In this case, by using the lower bound in Theorem 3.10 we get that

$$
\begin{aligned}
r(t, x, y) & \geq C_{1} \int_{0}^{|x-y|^{2}} s^{-d / 2} e^{-\frac{C_{2}|x-y|^{2}}{s}} \mu(t, s) d s \\
& =C_{1} \int_{0}^{|x-y|^{2}} s^{-d / 2} e^{-\frac{C_{2}|x-y|^{2}}{s}} t^{-2 / \alpha} \mu\left(1, t^{-2 / \alpha} s\right) d s \\
& =C_{1} t^{-d / \alpha} \int_{0}^{\frac{|x-y|^{2}}{t^{2 / \alpha}}} u^{-d / 2} e^{-\frac{C_{2}}{u} \frac{|x-y|^{2}}{t^{2 / \alpha}}} \mu(1, u) d u \\
& \geq C_{1} t^{-d / \alpha} \int_{\frac{|x-y|^{2}}{2 t^{2 / \alpha}}}^{\frac{|x-y|^{2}}{t^{2 / \alpha}}} u^{-d / 2} e^{-\frac{C_{2}}{u} \frac{|x-y|^{2}}{t^{2 / \alpha}}} \mu(1, u) d u \\
& \geq c_{10} t^{-d / \alpha}\left(\frac{|x-y|^{2}}{t^{2 / \alpha}}\right)\left(\frac{|x-y|^{2}}{t^{2 / \alpha}}\right)^{-d / 2}\left(\frac{|x-y|^{2}}{t^{2 / \alpha}}\right)^{-\alpha / 2-1} \\
& =c_{10} t^{-d / \alpha}\left(\frac{|x-y|^{2}}{t^{2 / \alpha}}\right)^{-\frac{d+\alpha}{2}} \geq c_{10} t^{-d / \alpha}\left(1+\frac{|x-y|^{2}}{t^{2 / \alpha}}\right)^{-\frac{d+\alpha}{2}},
\end{aligned}
$$

where in the fifth line above we used (4.2). 
(iii) $\rho(x) \rho(y)<1,|x-y|^{2} \leq \rho(x) \rho(y), \frac{|x-y|^{2}}{t^{2 / \alpha}} \leq 2 M, \frac{\rho(x) \rho(y)}{t^{2 / \alpha}} \geq 2 M$. In this case we have

$$
\begin{aligned}
r(t, x, y) & \geq C_{1} \int_{0}^{\rho(x) \rho(y)} s^{-d / 2} e^{-\frac{C_{2}|x-y|^{2}}{s}} \mu(t, s) d s \\
& \geq C_{1} t^{-d / \alpha} \int_{0}^{\frac{\rho(x) \rho(y)}{t^{2 / \alpha}}} u^{-d / 2} e^{-\frac{C_{2}}{u} \frac{|x-y|^{2}}{t^{2 / \alpha}}} \mu(1, u) d u \\
& \geq C_{1} t^{-d / \alpha} \int_{M}^{2 M} u^{-d / 2} e^{-\frac{2 C_{2} M}{u}} \mu(1, u) d u \geq c_{11} t^{-d / \alpha} .
\end{aligned}
$$

(iv) $\rho(x) \rho(y)<1,|x-y|^{2} \leq \rho(x) \rho(y), \frac{\rho(x) \rho(y)}{t^{2 / \alpha}} \leq 2 M$ and $\frac{1}{t^{2 / \alpha}} \geq 3 M$. In this case we have

$$
\begin{aligned}
r(t, x, y) \geq & C_{1} \int_{\rho(x) \rho(y)}^{1} \rho(x) \rho(y) s^{-(d+2) / 2} e^{-\frac{C_{2}|x-y|^{2}}{s}} \mu(t, s) d s \\
= & C_{1} \rho(x) \rho(y) t^{-\frac{d+2}{\alpha}} \int_{\frac{\rho(x) \rho(y)}{t^{2 / \alpha}}}^{\frac{1}{t^{2 / \alpha}}} u^{-(d+2) / 2} e^{-\frac{C_{2}}{u} \frac{|x-y|^{2}}{t^{2 / \alpha}}} \mu(1, u) d u \\
\geq & C_{1} \rho(x) \rho(y) t^{-\frac{d+2}{\alpha}} \int_{2 M}^{3 M} u^{-(d+2) / 2} e^{-\frac{2 C_{2} M}{u}} \mu(1, u) d u \\
\geq & c_{12} \rho(x) \rho(y) t^{-\frac{d+2}{\alpha}} \geq c_{13} \frac{\rho(x) \rho(y)}{\left(t^{2 / \alpha}+|x-y|^{2}\right) \wedge 1} t^{-\frac{d}{\alpha}} \\
& \times\left(1+\frac{|x-y|^{2}}{t^{2 / \alpha}}\right)^{-\frac{d+\alpha}{2}} .
\end{aligned}
$$

(v) $\rho(x) \rho(y)<1,|x-y|^{2} \leq \rho(x) \rho(y), \frac{\rho(x) \rho(y)}{t^{2 / \alpha}} \leq 2 M$ and $\frac{1}{t^{2 / \alpha}} \leq 3 M$. In this case we have

$$
\begin{aligned}
r(t, x, y) \geq & C_{1} \int_{1}^{\infty} \rho(x) \rho(y) s^{-d / 2} e^{-\frac{C_{2}|x-y|^{2}}{s}} \mu(t, s) d s \\
= & C_{1} \rho(x) \rho(y) t^{-\frac{d}{\alpha}} \int_{\frac{1}{t^{2 / \alpha}}}^{\infty} u^{-d / 2} e^{-\frac{C_{2}}{u} \frac{|x-y|^{2}}{t^{2 / \alpha}}} \mu(1, u) d u \\
\geq & C_{1} \rho(x) \rho(y) t^{-\frac{d}{\alpha}} \int_{3 M}^{\infty} u^{-d / 2} e^{-\frac{2 C_{2} M}{u}} \mu(1, u) d u \\
\geq & c_{14} \rho(x) \rho(y) t^{-\frac{d}{\alpha}} \geq c_{15} \frac{\rho(x) \rho(y)}{\left(t^{2 / \alpha}+|x-y|^{2}\right) \wedge 1} t^{-\frac{d}{\alpha}} \\
& \times\left(1+\frac{|x-y|^{2}}{t^{2 / \alpha}}\right)^{-\frac{d+\alpha}{2}} .
\end{aligned}
$$


(vi) $\rho(x) \rho(y)<1,|x-y|^{2}>\rho(x) \rho(y), \frac{\rho(x) \rho(y)}{t^{2 / \alpha}} \geq 2 M$ and $\frac{|x-y|^{2}}{t^{2 / \alpha}} \leq 3 M$. In this case we have

$$
\begin{aligned}
r(t, x, y) & \geq C_{1} \int_{0}^{\rho(x) \rho(y)} s^{-d / 2} e^{-\frac{C_{2}|x-y|^{2}}{s}} \mu(t, s) d s \\
& =C_{1} t^{-d / \alpha} \int_{0}^{\frac{\rho(x) \rho(y)}{t^{2 / \alpha}}} u^{-d / 2} e^{-\frac{C_{2}}{u} \frac{|x-y|^{2}}{t^{2 / \alpha}}} \mu(1, u) d u \\
& \geq C_{1} t^{-d / \alpha} \int_{0}^{2 M} u^{-d / 2} e^{-\frac{3 C_{2} M}{u}} \mu(1, u) d u \geq c_{16} t^{-d / \alpha} .
\end{aligned}
$$

(vii) $\rho(x) \rho(y)<1,|x-y|^{2}>\rho(x) \rho(y), \frac{\rho(x) \rho(y)}{t^{2 / \alpha}} \leq 2 M$, and $\frac{|x-y|^{2}}{t^{2 / \alpha}} \leq 3 M$. In this case we have

$$
\begin{aligned}
r(t, x, y) \geq & C_{1} \int_{\rho(x) \rho(y)}^{\infty} \rho(x) \rho(y) s^{-(d+2) / 2} e^{-\frac{C_{2}|x-y|^{2}}{s}} \mu(t, s) d s \\
= & C_{1} \rho(x) \rho(y) t^{-\frac{d+2}{\alpha}} \int_{\frac{\rho(x) \rho(y)}{t^{2 / \alpha}}}^{\infty} u^{-(d+2) / 2} e^{-\frac{C_{2}}{u} \frac{|x-y|^{2}}{t^{2 / \alpha}}} \mu(1, u) d u \\
\geq & C_{1} \rho(x) \rho(y) t^{-\frac{d+2}{\alpha}} \int_{2 M}^{\infty} u^{-(d+2) / 2} e^{-\frac{3 C_{2} M}{u}} \mu(1, u) d u \\
\geq & c_{17} \rho(x) \rho(y) t^{-\frac{d+2}{\alpha}} \geq c_{17} \frac{\rho(x) \rho(y)}{t^{2 / \alpha}+|x-y|^{2}} t^{-\frac{d}{\alpha}} \\
& \times\left(1+\frac{|x-y|^{2}}{t^{2 / \alpha}}\right)^{-\frac{d+\alpha}{2}},
\end{aligned}
$$

and

$$
\begin{aligned}
r(t, x, y) & \geq C_{1} \int_{\rho(x) \rho(y)}^{\infty} \rho(x) \rho(y) s^{-d / 2} e^{-\frac{C_{2}|x-y|^{2}}{s}} \mu(t, s) d s \\
& =C_{1} \rho(x) \rho(y) t^{-\frac{d}{\alpha}} \int_{\frac{\rho(x) \rho(y)}{t^{2 / \alpha}}}^{\infty} u^{-d / 2} e^{-\frac{C_{2}}{u} \frac{|x-y|^{2}}{t^{2 / \alpha}}} \mu(1, u) d u \\
& \geq C_{1} \rho(x) \rho(y) t^{-\frac{d}{\alpha}} \int_{2 M}^{\infty} u^{-d / 2} e^{-\frac{3 C_{2} M}{u}} \mu(1, u) d u \\
& \geq c_{18} \rho(x) \rho(y) t^{-\frac{d}{\alpha}} \geq c_{18} \rho(x) \rho(y) t^{-\frac{d}{\alpha}}\left(1+\frac{|x-y|^{2}}{t^{2 / \alpha}}\right)^{-\frac{d+\alpha}{2}}
\end{aligned}
$$

Combining the above we get that in this case

$$
r(t, x, y) \geq c_{19} \frac{\rho(x) \rho(y)}{\left(t^{2 / \alpha}+|x-y|^{2}\right) \wedge 1} t^{-\frac{d}{\alpha}}\left(1+\frac{|x-y|^{2}}{t^{2 / \alpha}}\right)^{-\frac{d+\alpha}{2}}
$$


(viii) $\rho(x) \rho(y)<1,|x-y|^{2}>\rho(x) \rho(y)$ and $\frac{|x-y|^{2}}{t^{2 / \alpha}} \geq 3 M$. In this case we have

$$
\begin{aligned}
r(t, x, y) \geq & C_{1} \int_{|x-y|^{2}}^{\infty} \rho(x) \rho(y) s^{-d / 2} e^{-\frac{C_{2}|x-y|^{2}}{s}} \mu(t, s) d s \\
\geq & C_{1} \rho(x) \rho(y) t^{-\frac{d}{\alpha}} \int_{\frac{|x-y|^{2}}{t^{2 / \alpha}}}^{\frac{2|x-y|^{2}}{t^{2 / \alpha}}} u^{-d / 2} e^{-\frac{C_{2}}{u} \frac{|x-y|^{2}}{t^{2 / \alpha}}} \mu(1, u) d u \\
\geq & c_{20} \rho(x) \rho(y) t^{-d / \alpha}\left(\frac{|x-y|^{2}}{t^{2 / \alpha}}\right)\left(\frac{|x-y|^{2}}{t^{2 / \alpha}}\right)^{-d / 2} \\
& \times\left(\frac{|x-y|^{2}}{t^{2 / \alpha}}\right)^{-\alpha / 2-1} \\
= & c_{20} \rho(x) \rho(y) t^{-d / \alpha}\left(\frac{|x-y|^{2}}{t^{2 / \alpha}}\right)^{-\frac{d+\alpha}{2}} \\
\geq & c_{20} \rho(x) \rho(y) t^{-d / \alpha}\left(1+\frac{|x-y|^{2}}{t^{2 / \alpha}}\right)^{-\frac{d+\alpha}{2}},
\end{aligned}
$$

and

$$
\begin{aligned}
r(t, x, y) \geq & C_{1} \int_{|x-y|^{2}}^{\infty} \rho(x) \rho(y) s^{-(d+2) / 2} e^{-\frac{C_{2}|x-y|^{2}}{s}} \mu(t, s) d s \\
\geq & C_{1} \rho(x) \rho(y) t^{-\frac{d+2}{\alpha}} \int_{\frac{|x-y|^{2}}{t^{2 / \alpha}}}^{\frac{2|x-y|^{2}}{t^{2 / \alpha}}} u^{-(d+2) / 2} e^{-\frac{C_{2}}{u} \frac{|x-y|^{2}}{t^{2 / \alpha}}} \mu(1, u) d u \\
\geq & c_{21} \rho(x) \rho(y) t^{-(d+2) / \alpha}\left(\frac{|x-y|^{2}}{t^{2 / \alpha}}\right)\left(\frac{|x-y|^{2}}{t^{2 / \alpha}}\right)^{-(d+2) / 2} \\
& \times\left(\frac{|x-y|^{2}}{t^{2 / \alpha}}\right)^{-\alpha / 2-1} \\
= & c_{21} \rho(x) \rho(y) t^{-(d+2) / \alpha}\left(\frac{|x-y|^{2}}{t^{2 / \alpha}}\right)^{-\frac{d+2+\alpha}{2}} \\
\geq & c_{21} \rho(x) \rho(y) t^{-d / \alpha}\left(1+\frac{|x-y|^{2}}{t^{2 / \alpha}}\right)^{-\frac{d+\alpha}{2}} \\
= & c_{21} \frac{\rho(x) \rho(y)}{t^{2 / \alpha}+|x-y|^{2}} t^{-\frac{d}{\alpha}}\left(1+\frac{|x-y|^{2}}{t^{2 / \alpha}}\right)^{-\frac{d+\alpha}{2}}
\end{aligned}
$$

where we used (4.2) in the third lines of both displays above. Combining the above we get that in this case

$$
r(t, x, y) \geq c_{22} \frac{\rho(x) \rho(y)}{\left(t^{2 / \alpha}+|x-y|^{2}\right) \wedge 1} t^{-\frac{d}{\alpha}}\left(1+\frac{|x-y|^{2}}{t^{2 / \alpha}}\right)^{-\frac{d+\alpha}{2}} .
$$

The proof of the lower bound is now complete. 
For bounded $C^{1,1}$ domains we have the following result.

Theorem 4.7. Suppose that $D$ is a bounded $C^{1,1}$ domain in $\mathbb{R}^{d}, d \geq 1$. For any $T_{1}>0$, there exist positive constants $C_{1}$ and $C_{2}$ such that for any $t \in\left(0, T_{1}\right]$ and $x, y \in D$,

$$
\begin{aligned}
& C_{1}\left(\frac{\rho(x) \rho(y)}{t^{2 / \alpha}+|x-y|^{2}} \wedge 1\right) t^{-\frac{d}{\alpha}}\left(1+\frac{|x-y|^{2}}{t^{2 / \alpha}}\right)^{-\frac{d+\alpha}{2}} \leq r(t, x, y) \\
& \leq C_{2}\left(\frac{\rho(x) \rho(y)}{t^{2 / \alpha}+|x-y|^{2}} \wedge 1\right) t^{-\frac{d}{\alpha}}\left(1+\frac{|x-y|^{2}}{t^{2 / \alpha}}\right)^{-\frac{d+\alpha}{2}} .
\end{aligned}
$$

Proof. The upper bound is actually valid for all $t>0$. To get this upper bound, we just need to use Theorem 3.1. So we only deal with the lower bound.

Let $R$ be the diameter of $D$. Without loss of generality we may assume that $R^{2}<T_{1} / 2$. In the remainder of this proof $C_{2}$ stands for the constant $C_{2}$ in Theorem 3.9. We deal with the following separate cases.

(i) $|x-y|^{2} \leq \rho(x) \rho(y)$ and $\frac{|x-y|^{2}}{t^{2 / \alpha}} \geq 2 M$. Using exactly the same argument as in case (ii) of the proof of the theorem above we get

$$
r(t, x, y) \geq c_{1} t^{-d / \alpha}\left(1+\frac{|x-y|^{2}}{t^{2 / \alpha}}\right)^{-\frac{d+\alpha}{2}} .
$$

The only difference is that we use Theorem 3.9 for $T=T_{1}$ here instead of Theorem 3.10.

(ii) $|x-y|^{2} \leq \rho(x) \rho(y), \frac{\rho(x) \rho(y)}{t^{2 / \alpha}} \geq 2 M$ and $\frac{|x-y|^{2}}{t^{2 / \alpha}} \leq 2 M$. Using exactly the same argument as in case (iii) of the proof of the theorem above we get

$$
r(t, x, y) \geq c_{2} t^{-d / \alpha} .
$$

Again the only difference is that we use Theorem 3.9 for $T=T_{1}$ here instead of Theorem 3.10.

(iii) $|x-y|^{2} \leq \rho(x) \rho(y), \frac{\rho(x) \rho(y)}{t^{2 / \alpha}} \leq 2 M$ and $\frac{|x-y|^{2}}{t^{2 / \alpha}} \leq 2 M$. In this case, by using Theorem 3.9 for $T=4 T_{1}^{2 / \alpha} M$, we get

$$
\begin{aligned}
r(t, x, y) & \geq c_{3} \int_{\rho(x) \rho(y)}^{4 T_{1}^{2 / \alpha} M} \rho(x) \rho(y) s^{-(d+2) / 2} e^{-\frac{C_{2}|x-y|^{2}}{s}} \mu(t, s) d s \\
& =c_{3} \rho(x) \rho(y) t^{-\frac{d+2}{\alpha}} \int_{\frac{\rho(x) \rho(y)}{t^{2 / \alpha}}}^{\frac{4 T_{1}^{2 / \alpha} M}{t^{2 / \alpha}}} u^{-(d+2) / 2} e^{-\frac{C_{2}}{u} \frac{|x-y|^{2}}{t^{2 / \alpha}}} \mu(1, u) d u \\
& \geq c_{4} \rho(x) \rho(y) t^{-\frac{d+2}{\alpha}} \int_{2 M}^{\frac{4 T_{1}^{2 / \alpha} M}{t^{2 / \alpha}}} u^{-(d+2) / 2} e^{-\frac{2 C_{2} M}{u}} \mu(1, u) d u \\
& \geq c_{5} \rho(x) \rho(y) t^{-\frac{d+2}{\alpha}} .
\end{aligned}
$$


(iv) $|x-y|^{2}>\rho(x) \rho(y)$ and $\frac{|x-y|^{2}}{t^{2 / \alpha}} \geq M$. In this case, by using Theorem 3.9 for $T=T_{1}$, we get

$$
\begin{aligned}
r(t, x, y) \geq & c_{3} \int_{\rho(x) \rho(y)}^{T_{1}} \rho(x) \rho(y) s^{-(d+2) / 2} e^{-\frac{C_{2}|x-y|^{2}}{s}} \mu(t, s) d s \\
= & c_{3} \rho(x) \rho(y) t^{-\frac{d+2}{\alpha}} \int_{\frac{\rho(x) \rho(y)}{t^{2 / \alpha}}}^{\frac{T_{1}}{t^{2 / \alpha}}} u^{-(d+2) / 2} e^{-\frac{C_{2}}{u} \frac{|x-y|^{2}}{t^{2 / \alpha}}} \mu(1, u) d u \\
\geq & c_{6} \rho(x) \rho(y) t^{-\frac{d+2}{\alpha}} \int_{\frac{\rho(x) \rho(y)}{t^{2 / \alpha}}}^{\frac{2 R^{2}}{t^{2 / \alpha}}} u^{-(d+2) / 2} e^{-\frac{c_{2}}{u} \frac{|x-y|^{2}}{t^{2 / \alpha}}} \mu(1, u) d u \\
\geq & c_{6} \rho(x) \rho(y) t^{-\frac{d+2}{\alpha}} \int_{\frac{|x-y|^{2}}{t^{2 / \alpha}}}^{\frac{2|x-y|^{2}}{t^{2 / \alpha}}} u^{-(d+2) / 2} e^{-\frac{C_{2}}{u} \frac{|x-y|^{2}}{t^{2 / \alpha}}} \mu(1, u) d u \\
\geq & c_{7} \rho(x) \rho(y) t^{-\frac{d+2}{\alpha}}\left(\frac{|x-y|^{2}}{t^{2 / \alpha}}\right)\left(\frac{|x-y|^{2}}{t^{2 / \alpha}}\right)^{-(d+2) / 2} \\
& \times\left(\frac{|x-y|^{2}}{t^{2 / \alpha}}\right)^{-\alpha / 2-1} \\
= & c_{7} \rho(x) \rho(y) t^{-\frac{d+2}{\alpha}}\left(\frac{|x-y|^{2}}{t^{2 / \alpha}}\right)^{-\frac{d+2+\alpha}{2}} \\
\geq & c_{7} \rho(x) \rho(y) t^{-\frac{d+2}{\alpha}}\left(1+\frac{|x-y|^{2}}{t^{2 / \alpha}}\right)^{-\frac{d+2+\alpha}{2}},
\end{aligned}
$$

where in the fifth line above we used (4.2).

(v) $|x-y|^{2}>\rho(x) \rho(y)$ and $\frac{|x-y|^{2}}{t^{2 / \alpha}} \leq M$. In this case, by using Theorem 3.9 for $T=4 T_{1}^{2 / \alpha} M$, we get

$$
\begin{aligned}
r(t, x, y) & \geq c_{3} \int_{\rho(x) \rho(y)}^{4 T_{1}^{2 / \alpha} M} \rho(x) \rho(y) s^{-(d+2) / 2} e^{-\frac{C_{2}|x-y|^{2}}{s}} \mu(t, s) d s \\
& =c_{3} \rho(x) \rho(y) t^{-\frac{d+2}{\alpha}} \int_{\frac{\rho(x) \rho(y)}{t^{2 / \alpha}}}^{\frac{4 T_{1}^{2 / \alpha} M}{t^{2 / \alpha}}} u^{-(d+2) / 2} e^{-\frac{C_{2}}{u} \frac{|x-y|^{2}}{t^{2 / \alpha}}} \mu(1, u) d u \\
& \geq c_{8} \rho(x) \rho(y) t^{-\frac{d+2}{\alpha}} \int_{\frac{R^{2}}{t^{2 / \alpha}}}^{\frac{4 T_{1}^{2 / \alpha} M}{t^{2 / \alpha}}} u^{-(d+2) / 2} e^{-\frac{C_{2} M}{u}} \mu(1, u) d u \\
& \geq c_{9} \rho(x) \rho(y) t^{-\frac{d+2}{\alpha}} .
\end{aligned}
$$

Combining the five cases above we get the desired lower bound.

Remark 4.8. One could also use the results of the two theorems above to get the Green function estimates in Theorems 4.1 and 4.3. 
Acknowledgements. I would like to thank Zoran Vondracek for his helpful comments on the first version of this paper. I would also like to thank the referees for their detailed and helpful comments on the first version of this paper.

\section{References}

1. Blumenthal, R.M., Getoor, R.K.: Some theorems on stable processes. Trans. Am. Math. Soc. 95, 263-273 (1960)

2. Blumenthal, R.M., Getoor, R.K.: Markov processes and potential theory. Academic Press, New York, 1968

3. Bouleau, N.: Quelques résultats probabilistes sur la subordination au sens de Bochner. In: Seminar on Potential Theory, Paris, No. 7, Lecture Notes in Math., 1061, Springer, Berlin, 1984, pp. 54-81

4. Chen, Z.-Q., Song, R.: Estimates on Green functions and Poisson kernels for symmetric stable processes. Math. Ann. 312, 465-501 (1998)

5. Chen,Z.-Q., Song, R.: General gauge and conditional gauge theorems. Ann. Probab. 30, 1313-1339 (2002)

6. Chung, K.L., Zhao, Z.: From Brownian motion to Schrödinger equations. Springer, Berlin, 1995

7. Davies, E.B.: Heat kernels and spectral theory. Cambridge University Press, Cambridge, 1989

8. Fabes, E.B., Garofalo, N., Salsa, S.: A backward Harnack inequality and Fatou theorem for nonnegative solutions of parabolic equations. Illinois J. Math. 30, 536-565 (1986)

9. Farkas, W., Jacob, N.: Sobolev spaces on non-smooth domains and Dirichlet forms related to subordinate reflecting diffusions. Math. Nachr. 224, 75-104 (2001)

10. Fukushima, M., Oshima, Y., Takeda, M.: Dirichlet forms and symmetric Markov processes. Walter De Gruyter, Berlin, 1994

11. Glover, J., Rao, M., Šikić, H., Song, R.: Г-potentials. In: Classical and modern potential theory and applications (Chateau de Bonas, 1993), Kluwer Acad. Publ., Dordrecht, 1994, pp. 217-232

12. Ikeda, N., Watanabe, S.: On some relations between the harmonic measure and the Lévy measure for a certain class of Markov processes. J. Math. Kyoto Univ. 2, 79-95 (1962)

13. Jacob, N., Schilling, R.: Some Dirichlet spaces obtained by subordinate reflected diffusions. Rev. Mat. Iberoamericana 15, 59-91 (1999)

14. Kulczycki, T.: Properties of Green function of symmetric stable processes. Probab. Math. Statist. 17, 339-364 (1997)

15. Miyake, A.: The subordination of Lévy system for Markov processes. Proc. Japan Acad. 45, 601-604 (1969)

16. Pazy, A.: Semigroups of linear operators and applications to partial differential equations. Springer-Verlag, New York, 1983

17. Schilling, R.L.: On the domain of the generator of a subordinate semigroup. In: J. Král et al. (eds.), Potential theory- ICPT 94. Proceedings Intl. Conf. Potential Theory, Kouty (CR), 1994 de Gruyter. 1996, pp. 449-462

18. Song, R., Vondracek, Z.: Potential theory of subordinate killed Brownian motion in a domain. Probab. Theory Relat. Fields. 135, 578-592 (2003)

19. Yosida, K.: Functional analysis. 6th Edition, Springer-Verlag, Berlin, 1980

20. Zhang, Q.S.: The boundary behavior of heat kernels of Dirichlet Laplacians. J. Diff. Eqs. 182, 416-430 (2002)

21. Zhang, Q.S.: The global behavior of heat kernels in exterior domains. J. Funct. Anal. 200, 160-176 (2003)

22. Zolotarev, V.M.: One-dimensional stable distributions. Am. Math. Soc. Providence, RI, 1986 